\title{
Arteriolar and venular patterning in retinas of mice selectively expressing VEGF isoforms
}

\author{
Ingeborg Stalmans, ${ }^{1}$ Yin-Shan Ng, ${ }^{2}$ Richard Rohan, ${ }^{3}$ Marcus Fruttiger, ${ }^{4}$ Ann Bouché, \\ Ali Ÿuce, ${ }^{5}$ Hajime Fujisawa, ${ }^{6}$ Bart Hermans, ${ }^{1}$ Moshe Shani, ${ }^{7}$ Sandra Jansen, ${ }^{1}$ Dan Hicklin, ${ }^{8}$ \\ David J. Anderson, ${ }^{9}$ Tom Gardiner ${ }^{10}$ Hans-Peter Hammes, ${ }^{11}$ Lieve Moons, ${ }^{1}$ \\ Mieke Dewerchin, ${ }^{1}$ Désiré Collen, ${ }^{1}$ Peter Carmeliet, ${ }^{1}$ and Patricia A. D’Amore ${ }^{2}$ \\ ${ }^{1}$ The Center for Transgene Technology and Gene Therapy, Flanders Interuniversity Institute for Biotechnology, \\ Catholic University Leuven, Leuven, Belgium \\ ${ }^{2}$ The Schepens Eye Research Institute Department of Pathology and Ophthalmology, Harvard Medical School, \\ Boston, Massachusetts, USA \\ ${ }^{3}$ Children's Hospital, Harvard Medical School, Boston, Massachusetts, USA \\ ${ }^{4}$ Wolfson Institute for Biomedical Research, University College London, London, United Kingdom \\ ${ }^{5}$ Third Department of Internal Medicine, Justus Liebig University, Giessen, Germany \\ ${ }^{6}$ Department of Molecular Biology, Nagoya University, Nagoya, Japan \\ ${ }^{7}$ Institute of Animal Science, The Volcani Center, Bet Dagan, Israel \\ ${ }^{8}$ Department of Immunology, ImClone Systems Incorporated, New York, New York, USA \\ ${ }^{9}$ Division of Biology and Howard Hughes Medical Institute, California Institute of Technology, Pasadena, California, USA \\ ${ }^{10}$ Ophthalmology and Vision Science, Institute of Clinical Science, Queen's University, Belfast, Ireland \\ ${ }^{11}$ Medical Department, University Clinic, Mannheim, Germany
}

Address correspondence to: Peter Carmeliet, Center for Transgene Technology and Gene Therapy, Flanders Interuniversity Institute for Biotechnology, Catholic University Leuven, Campus Gasthuisberg, Herestraat 49, B-3000, Leuven, Belgium. Phone: 32-16-34-57-72; Fax: 32-16-34-59-90; E-mail: peter.carmeliet@med.kuleuven.ac.be.

Received for publication October 9, 2001, and accepted in revised form December 27, 2001.

\begin{abstract}
The murine $V E G F$ gene is alternatively transcribed to yield the $V_{E G F} F_{120}, V_{E G F} F_{164}$, and $V_{E G F} F_{188}$ isoforms, which differ in their potential to bind to heparan sulfate and neuropilin-1 and to stimulate endothelial growth. Here, their role in retinal vascular development was studied in mice selectively expressing single isoforms. $V E G F^{164 / 164}$ mice were normal, healthy, and had normal retinal angiogenesis. In contrast, $V E G F^{120 / 120}$ mice exhibited severe defects in vascular outgrowth and patterning, whereas $V E G F^{188 / 188}$ mice displayed normal venular outgrowth but impaired arterial development. It is noteworthy that neuropilin-1, a receptor for $\mathrm{VEGF}_{164}$, was predominantly expressed in retinal arterioles. These findings reveal distinct roles of the various VEGF isoforms in vascular patterning and arterial development in the retina.
\end{abstract}

J. Clin. Invest. 109:327-336 (2002). DOI:10.1172/JCI200214362.

\section{Introduction}

The essential role of VEGF in blood vessel formation in the embryo and in the adult is well established $(1,2)$. A single $V E G F$ gene gives rise, by alternative splicing, to multiple isoforms $\left(\mathrm{VEGF}_{121}, \mathrm{VEGF}_{165}\right.$, and $\mathrm{VEGF}_{189}$ in humans versus $\mathrm{VEGF}_{120}, \mathrm{VEGF}_{164}$, and $\mathrm{VEGF}_{188}$ in the mouse) that differ in molecular mass, solubility, and receptor binding (3). VEGF 120 lacks exons 6 and 7 , encoding extracellular matrix binding structures, and is therefore the most soluble. VEGF $_{188}$ contains all exons and avidly binds to the cell surface and extracellular matrix. VEGF 164 lacks only exon 6 and has intermediate properties. The VEGF isoforms bind to several receptors: VEGFR-1 (Flt-1), VEGFR-2 (Flk-1), and neuropilin1 (NP-1) (3). NP-1 is a semaphorin receptor involved in neuron guidance. As a $\mathrm{VEGF}_{164}$-specific coreceptor for VEGFR-2, NP-1 also affects angiogenesis (4) and enhances the angiogenic activity of $\mathrm{VEGF}_{164}(5)$

To define the differential role of the VEGF isoforms in vivo, mice expressing single VEGF isoforms were gen- erated. Impaired myocardial angiogenesis has been demonstrated in $V E G F^{120 / 120}$ mice (expressing VEGF ${ }_{120}$ ) (6). Here we report that loss of VEGF ${ }_{164}$ (in VEGF ${ }^{120 / 120}$ and $V E G F^{188 / 188}$ mice) impaired retinal arterial development, whereas loss of $\mathrm{VEGF}_{164}$ and $\mathrm{VEGF}_{188}$ (in $V E G F^{120 / 120}$ mice) led to dysregulated vessel outgrowth and patterning in the retina. These observations suggest possible mechanisms for the distinct roles of the VEGF isoforms in retinal vascular patterning and arterial endothelial cell specification.

\section{Methods}

Generation of transgenic mice. Targeted mutagenesis was achieved by homologous and Cre/lox $P$-mediated sitespecific recombination in embryonic stem (ES) cells. The strategy to generate $V E G F^{120 / 120}$ mice (deletion of exons 6 and 7) has been described previously (6). Targeting vectors used to generate $V E G F^{164 / 164}$ mice and $V E G F^{188 / 188}$ mice were constructed by replacing the genomic sequence with a cDNA containing the fused 

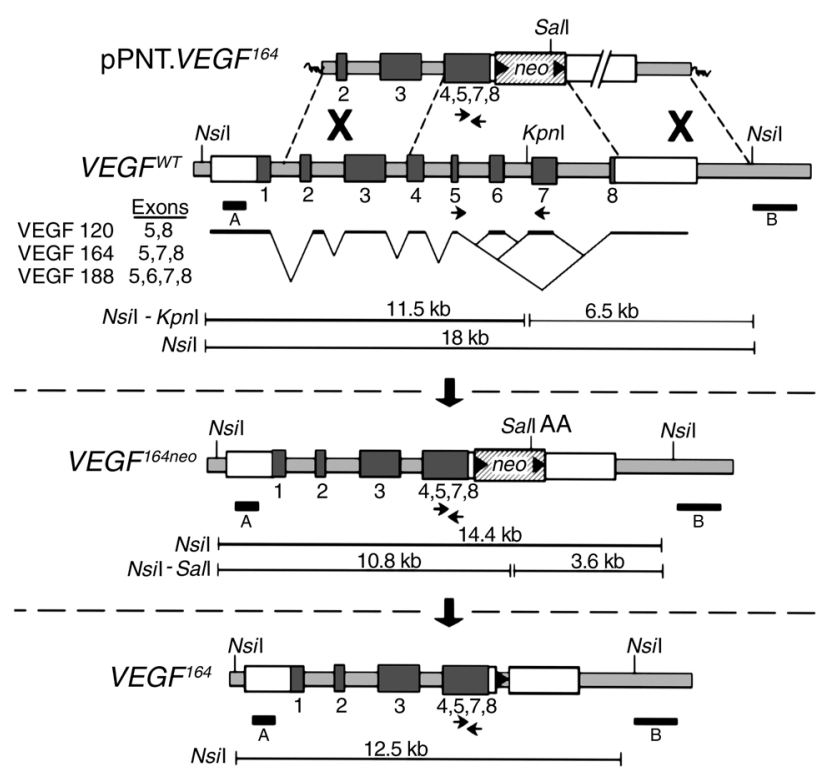

\section{Figure 1}

Targeting strategy. Modification of the VEGF gene to generate the $V E G F^{164}$ targeting vector. Top: Targeting vector pPNT.VEGF ${ }^{164}$ and wild-type VEGF allele (VEGFWT). Middle: Homologously recombined VEGF 164 allele $\left(V E G F^{164 n e o}\right)$. Bottom: Modified VEGF ${ }^{164}$ allele after Cre excision of the lox P-flanked neo cassette.

exons $4,5,7$, and 8 (for $V E G F_{164}$; Figure 1 ) or 4 to 8 (for $\left.V E G F_{188}\right)$. A lox $P$-flanked neomycin phosphotransferase (neo) cassette was placed in the $3^{\prime}$ UTR to allow positive selection. After electroporation, recombinant ES cell clones were identified by Southern blot analysis of genomic DNA (6) and transfected with a Cre expression plasmid to excise the lox $P$-flanked neo cassette. Cre-excised ES cell clones were used for diploid aggregation, yielding $V E G F^{+/ 188}$ mice. ES cell clones carrying a mutant $V E G F_{164}$ allele and neo were used for diploid aggregation, and the resulting germline mice were intercrossed with a mouse line expressing the Cre transgene under the activity of the phosphoglycerokinase (PGK) promoter to achieve in vivo excision of the neo cassette. The resulting $V E G F^{+/ 164}$ or $V E G F^{+/ 188}$ mice were bred to homozygosity. Genotyping was done by PCR using the following primers (in exon 5 and 7 , respectively): $5^{\prime}$ CCAAAGAAAGACAGGACAAAGCCAGAA $3^{\prime}$ and $5^{\prime}$ GCAAGTACGTTCGTTTAACTCAAGCTG $3^{\prime}$, resulting in PCR products of greater than $2 \mathrm{~kb}, 170 \mathrm{bp}$, and $230 \mathrm{bp}$ in $V E G F^{+/+}, V E G F^{164 / 164}$, and $V E G F^{188 / 188}$ mice, respectively. VEGF isoform-specific mice were intercrossed with mice expressing a $\beta$-galactosidase ( $\operatorname{Lac} Z$ ) gene under an ephrin-B2 promoter (7), a Tie1 promotor (8), or a promoter specific for pericytes and vascular smooth muscle cells (9).

Histology, whole-mount in situ bybridization, and retinal trypsin digest. All methods for immunohistochemistry and LacZ staining have been described $(6,7,10)$. Endothelial cells, vascular smooth muscle cells, and astrocytes, were labeled with biotinylated Bandeiraea simplicifolia (BS-1) Isolectin (L3759; Sigma-Aldrich NV/SA,
Bornem, Belgium) or anti-CD31 (557355; BD Biosciences, Erembodegem, Belgium), anti- $\alpha$-smooth muscle actin (labeled with FITC; F3777, Sigma-Aldrich NV/SA), and antiglial fibrillary acidic protein (Z0334; Dako SA, Trappes Cedex, France), respectively. Retinas were scanned with a Zeiss laser scanning microscope (LSM 510; Carl Zeiss Jena GmbH, Jena, Germany). For triple immunostainings, markers were combined with anti-VEGFR-1 (MF1) or anti-VEGFR-2 antiserum (457683; both from ImClone Systems Inc., New York, New York, USA), or rabbit anti-NP-1 antiserum (provided by H. Fujisawa, Department of Molecular Biology, Nagoya University, Nagoya, Japan; ref. 11). For electron microscopy, specimens were processed as described previously (6) and imaged on a Hitachi H7000 electron microscope. Whole-mount in situ hybridization was performed as described (12). The probes for ephrin-B2 and PDGFR $\beta$ were a gift from R. Klein, Department of Ophthalmology and Visual Sciences, University of Wisconsin Medical School, Madison, Wisconsin, USA (12) and C. Betsholtz, Department of Medical Biochemistry, Goteborg University, Goteborg, Sweden (13), respectively. The templates for NP-1 were obtained by reverse transcription of RNA from 6-day-old mouse retinae and PCR amplification of specific gene fragments using nested primer pairs (5' CCAAGCTCCGGAACCCTACCA $3^{\prime}$, $5^{\prime}$ CCTTGCGCTTGCTGTCATCCAT 3', 5' TCAGAACTATACAGCACCTACT 3', 5' TGAATGATGACACCTCTTACTA 3'). Immunolabeling with anticollagen type IV (Biogenesis Ltd., Poole, United Kingdom) was performed after in situ hybridization. Pericyte density was determined on pepsin-trypsin-digested preparations (14) and expressed per retinal area. The endothelial to pericyte ratio was quantitated using retinal image analysis.

\section{Results}

Targeting of VEGF isoform-specific alleles. The VEGF gene is alternatively spliced to the $\mathrm{VEGF}_{120}$ isoform (lacking exon 6 and 7), the $\mathrm{VEGF}_{164}$ isoform (lacking exon 6), and the $\mathrm{VEGF}_{188}$ isoform (encoded by all eight exons). Generation of mice selectively expressing single VEGF isoforms was achieved by Cre/lox P-mediated recombination in ES cells, using a "knock-in" strategy (see Methods; Figure 1). Quantitative RT-PCR analysis of brain RNA confirmed the selective expression of the respective VEGF isoform transcripts in each of these genotypes. Only the $V E G F_{164}$ isoform was detected in $V E G F^{164 / 164}$ mice $\left(340 \pm 46 V E G F_{164}\right.$ mRNA copies per $1,000$ hprt copies, mean $\pm \mathrm{SD}, n=5)$ and the $V E G F_{188}$ isoform in $V E G F^{188 / 188}$ mice $\left(770 \pm 210 V E G F_{188}\right.$ transcript copies per 1,000 hprt copies, $n=5)$, while the other isoforms were undetectable $\left(<0.3 V E G F_{164}\right.$ or $V E G F_{188}$ copies per 1,000 hprt copies). Total VEGF transcript levels of the VEGF isoforms were comparable to those in $V E G F^{+/+}$mice (total $V E G F$ mRNA copies per 1,000 hprt copies: $250 \pm 99$ in $V E G F^{+/+}, 230 \pm 75$ in $V E G F^{164 / 164}$, and $400 \pm 120$ in $V E G F^{188 / 188}$ mice; $n=5, P=$ not significant [NS]); the data in the VEGF ${ }^{120 / 120}$ mice have been reported previously (6). 
Inheritance and viability of VEGF-isoform mice. VEGF ${ }^{+/ 120}$, $V E G F^{+/ 164}$, and $V E G F^{+/ 188}$ mice were normal, healthy, and had a normal life span. Offspring of $V E G F^{+/ 120}$ breeding pairs were born at a normal Mendelian frequency, but half of $V E G F^{120 / 120}$ neonates died shortly after birth because of cardiorespiratory distress (6). The other $V E G F^{120 / 120}$ mice died within 2 weeks after birth, in part due to impaired myocardial angiogenesis resulting in cardiac failure (6).

$V E G F^{164 / 164}$ mice were born at a normal Mendelian frequency. Of 366 live neonates born from $V E G F^{+/ 164}$ breeding pairs, $25 \%$ were $V E G F^{+/+}, 51 \%$ were $V E G F^{+/ 164}$, and $24 \%$ were $V E G F^{164 / 164}$. They gained weight normally $\left(32 \pm 6 \mathrm{~g}\right.$ in $V E G F^{+/+}$mice, $n=8$, versus $32 \pm 4 \mathrm{~g}$ in $V E G F^{164 / 164}$ mice, $n=23$, at 7 weeks; $P=\mathrm{NS}$ ), were fertile (litters per breeding pair during 4 months: $4.8 \pm 1.3$ for $V E G F^{+/+}$mice, $n=4$, versus $4.8 \pm 0.5$ for $V_{E G F}{ }^{164 / 164}$ mice, $n=4 ; P=\mathrm{NS})$, and produced litters with normal size (11 \pm 3 pups for $V E G F^{+/+}$litters, $n=35$, versus $10 \pm 3$ for $V E G F^{164 / 164}$ litters, $\left.n=58 ; P=\mathrm{NS}\right)$.

$V E G F^{188 / 188}$ mice were underrepresented at birth; out of 73 live-born pups, 24\% were $V E G F^{+/+}$, 64\% were $V E G F^{+/ 188}$, and only $12 \%$ were $V E G F^{188 / 188}$, indicating that a fraction of the VEGF $F^{188 / 188}$ mice died in utero $(P<0.05$ for observed vs. expected). Surviving VEGF ${ }^{188 / 188}$ mice gained less weight than $V E G F^{+/+}$mice at 7 weeks of age $\left(32 \pm 6 \mathrm{~g}\right.$ in $V E G F^{+/+}$mice, $n=8$, versus $22 \pm 3 \mathrm{~g}$ in $V E G F^{188 / 188}$ mice, $\left.n=16 ; P<0.05\right)$. VEGF $F^{188 / 188}$ breeding pairs were less fertile (number of litters per breeding pair during 4 months: $4.8 \pm 0.5$ for $V E G F^{+/+}$mice, $n=4$, versus $3.7 \pm 0.6$ for $V E G F^{188 / 188}$ mice, $\left.n=3 ; P<0.05\right)$ and had smaller litter sizes $\left(11 \pm 3\right.$ pups for $V E G F^{+/+}$litters, $n=35$, versus $7 \pm 2$ pups for $V E G F^{188 / 188}$ litters, $n=53$; $\left.P<0.05\right)$.

Retinal vascular development in wild-type mice. Retinal vascularization was monitored by immunostaining of whole-mount preparations for CD31/PECAM or lectin for endothelial cells (ECs), smooth muscle alpha actin (SMA) for vascular smooth muscle cells (vSMCs) and pericytes (PCs), and GFAP for astrocytes (ACs). To study recruitment of periendothelial cells (PECs), VEGF-isoform mice were intercrossed with mice expressing $\mathrm{LacZ}$ in PECs (PEC ${ }^{\mathrm{LacZ}}$ mice) (9). Arterial specification of ECs was examined by in situ hybridization for ephrin-B2 and by intercrossing VEGF isoform mice with mice harboring a $L a c Z$ gene under the control of the endogenous ephrin-B2 promoter (EB2 ${ }^{\mathrm{LacZ}}$ mice) (7).
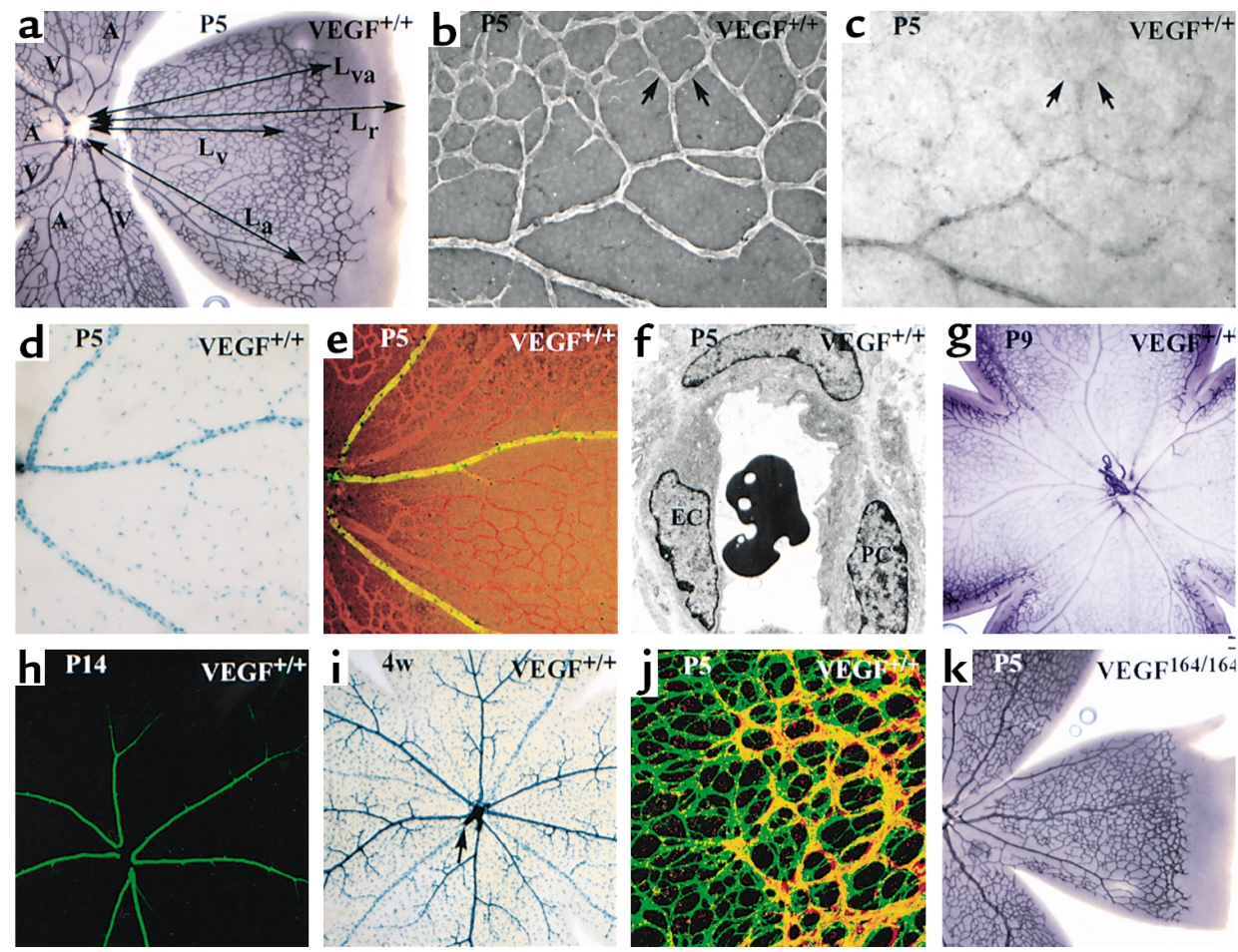

\section{Figure 2}

Retinal vascular development in $V E G F^{+/+}$and VEGF $164 / 164$ mice. (a) PECAM staining in $V E G F^{+/+}$at P5. Capillaries reach two-thirds to threequarters of the retinal radius. Arterioles (A) are thin and have a capillary-free zone. Venules $(\mathrm{V})$ are broader and lack a capillary-free zone. $L a$, arteriolar length; Lv, venular length; Lr, radius of retina; Lva, radius of vascular bed. (b and c) In situ hybridization for ephrin-B2 (EB2) (b) with or (c) without collagen type IV fluorescent immunostaining on VEGF ${ }^{+/+}$retina at P5 (EB2/CollIV). EB2 expression is confined to morphologically defined arterioles (see above) and extends into arterial capillaries (arrows). (d and e) Triple staining with LacZ (in PECs,

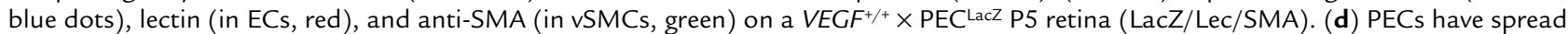
along all retinal vessels, whereas (e) only arterioles are SMA positive. (f) $E M$ of $V E G F^{+/+}$capillary at $\mathrm{P} 5$, which appears constricted. (g) $P E C A M$ staining on $V E G F^{+/+} \mathrm{P} 9$ retina. (h) $\mathrm{SMA}$ staining in $V E G F^{+/+}$at $\mathrm{P} 14$ is confined to arterioles and their side branches. (i) $V E G F^{+/+} \times \mathrm{PEC}^{\mathrm{LacZ}}$ retina at 4 weeks. Hyaloid arteries (HA) have regressed (HA remnant, arrow). (j) Double staining for lectin (red) and GFAP (in ACs, green) (Lec/GFAP). AC network in front of vasculature. (k) PECAM staining of VEGF $164 / 164$ retina at P5. Normal vascular development. 
Table 1

Characteristics of retinal vessels in VEGF-isoform mice

\begin{tabular}{lcccc}
\hline & VEGF & VEGF & & \\
& & & & \\
& & & \\
Arterioles & $5.7 \pm 1.0$ & $3.1 \pm 0.3^{\mathrm{A}}$ & $5.2 \pm 1.0$ & $2.8 \pm 1.5^{\mathrm{A}}$ \\
Number of arterioles/retina & $88 \pm 2$ & $35 \pm 9^{\mathrm{A}}$ & $89 \pm 10$ & $18 \pm 13^{\mathrm{A}}$ \\
La (\% of Lr) & $88 \pm 2$ & $53 \pm 6^{\mathrm{A}}$ & $90 \pm 6$ & $18 \pm 13^{\mathrm{A}}$ \\
La (\% of Lva) & $47 \pm 8.3$ & $41 \pm 9.8$ & $46 \pm 3.5$ & $22 \pm 0.4^{\mathrm{A}}$ \\
Width $(\mu \mathrm{m})$ & $23 \pm 0.9$ & $19 \pm 2^{\mathrm{A}}$ & $24 \pm 2$ & $16 \pm 2^{\mathrm{A}}$ \\
Number of PECs/mm artery & $5.7 \pm 1.0$ & $3.1 \pm 0.3^{\mathrm{A}}$ & $5.2 \pm 1.0$ & $2.8 \pm 1.5^{\mathrm{A}}$ \\
Number of vessels with capillary-free zone & + & + & ++ & + \\
SMA immunoreactivity & & & & \\
Venules & & & & \\
Number of venules per retina & $5.4 \pm 1.0$ & $3.1 \pm 0.3^{\mathrm{A}}$ & $5.1 \pm 1.0$ & $5.7 \pm 1.2$ \\
La (\% of Lr) & $79 \pm 1$ & $52 \pm 12^{\mathrm{A}}$ & $80 \pm 3$ & $76 \pm 6$ \\
La (\% of Lva) & $81 \pm 1$ & $80 \pm 4$ & $81 \pm 12$ & $79 \pm 6$ \\
Width $(\mu \mathrm{m})$ & $69 \pm 2$ & $76 \pm 15$ & $71 \pm 15$ & $74 \pm 9$ \\
Number of PECs/mm venule & $17 \pm 2$ & $14 \pm 4$ & $19 \pm 2$ & $19 \pm 2$ \\
SMA immunoreactivity & - & + & - & + \\
Capillaries & & & & \\
Number/mm & & & & \\
Width $(\mu \mathrm{m})$ & $190 \pm 18$ & $240 \pm 27^{\mathrm{A}}$ & $180 \pm 21$ & $310 \pm 21^{\mathrm{A}}$ \\
PECs/mm & $19 \pm 1.7$ & $29 \pm 4.2^{\mathrm{A}}$ & $18 \pm 1.6$ & $20 \pm 2.1$ \\
EC vs. PC ratio & $51 \pm 1$ & $39 \pm 6^{\mathrm{A}}$ & $51 \pm 3$ & $60 \pm 3^{\mathrm{A}}$ \\
SMA immunoreactivity & $5.3 \pm 0.2$ & $16 \pm 4^{\mathrm{A}}$ & $6.5 \pm 4$ & $7.2 \pm 1.4$ \\
Lva (\% of Lr) & - & + & - & + \\
& $99 \pm 0.5$ & $65 \pm 12^{\mathrm{A}}$ & $99 \pm 3$ & $99 \pm 1$
\end{tabular}

The data represent the mean \pm SD of 3 to 13 retinas at P9. Lengths of the arterioles and venules, as well as capillary outgrowth, are expressed as a percentage of the radius of the retina $(\mathrm{La} / \mathrm{Lr}, \mathrm{Lv} / \mathrm{Lr}, \mathrm{Lva} / \mathrm{Lv}$, respectively) and as a percentage of the radius of the vascular bed ( $L a / L v a$ and $L v / L v a$, respectively; Figure 2a). The diameter of the vessels is expressed in micrometers, the number of PCs along arterioles and venules in number per millimeter and per retinal area that is covered with capillaries (number $/ \mathrm{mm}^{2}$ ). ${ }^{A} P<0.05$ vs. control $\left(V E G F^{+/+}\right)$. La, arteriolar length; $L v$, venular length; $L r$, radius of retina; $L v a$, radius of vascular bed.

of PCs along the retinal capillaries, which contracted during fixation (Figure $2 \mathrm{f}$ ).

By P9, the vascular network had reached the periphery of the retina (Figure $2 \mathrm{~g}$ ). Arterioles and venules grew out over $88 \% \pm 4 \%$ and $79 \% \pm 6 \%$ of the retinal radius, respectively (Table 1). Venules were larger than arterioles, whereas capillaries had the smallest lumen (Table 1). On average, between five and six arterioles and venules were present per retina (Table 1). PECs covered arterioles, venules, and capillaries, resulting in a capillary EC-to$\mathrm{PC}$ ratio (EC/PC ratio) of $5: 1$ (Table 1). However, only the primary and secondary side branches of arterioles expressed detectable SMA levels, while the venules and capillaries remained negative at $\mathrm{P} 9$ and beyond (Figure $2 \mathrm{~h}$ ).

The hyaloid vasculature is present during development, but regresses once the devel-

Before birth, the murine retina is avascular and receives oxygen from the hyaloid and choroidal vessels. Postnatally, the increased retinal thickness and metabolism cause physiological hypoxia, which upregulates VEGF expression and initiates outgrowth of vessels from the optic disc (10). This results in the formation of a uniform capillary-like vascular labyrinth by postnatal day 2 (P2), which covers approximately $40 \%$ of the retina (not shown). Analysis of PEC ${ }^{\mathrm{LacZ}}$ mice revealed that these primitive vessels were already covered by PECs, which, however, did not express SMA (not shown). Using whole-mount in situ hybridization, ephrin-B2 was detectable in $50 \%$ of the retinal vessels (e.g., in future arterioles) in an alternating pattern (not shown), indicating that arteriovenous specification had already occurred at this early stage of vascular development.

By P5, the vascular bed covered approximately $70 \%$ of the retina and had remodeled into a more mature network of branching vessels, which could be morphologically distinguished as arterioles, venules, and capillaries (Figure 2a). Arterioles had a small lumen and were surrounded by a capillary-free zone (a result of capillary pruning), whereas venules were wider and embedded in capillary-rich regions. Ephrin-B2 was expressed in retinal arterioles but not in venules (Figure 2, b and c). Analysis of PEC ${ }^{\mathrm{LacZ}}$ mice revealed that PECs had spread along all vessels (Figure 2d), whereas expression of SMA was detectable in larger arterioles but not in venules or capillaries (Figure 2e). Transmission electron microscopy (EM) confirmed the presence oping retinal vasculature provides sufficient oxygen to the retina (15) (Figure 2i). Consistent with previous findings that the hyaloid vasculature contains only arteries (15), all hyaloid vessels in EB2 ${ }^{\mathrm{LacZ}}$ mice expressed ephrin-B2 (not shown). Hyaloid vessels were adjacent to the retina at P0 but did not make contact with retinal vessels and could easily be peeled away from the retina upon dissection of the retina from P0 up until their regression. They showed signs of regression by P9 and had disappeared by 4 weeks (Figure 2i).

Astrocyte development (ACs) have been implicated in guiding vessel outgrowth by producing a VEGF gradient (10). Whole-mount double immunofluorescent staining for GFAP and lectin revealed that spindleshaped ACs were spreading across the avascular retina at $\mathrm{P} 0.5$ and had reached the periphery of the retina by P2-3 (not shown). ACs in these avascular regions expressed abundant VEGF (not shown). At later times they became organized into a network of tubes wrapped around outgrowing vessels (Figure 2j). Once the vasculature had reached the retinal periphery (P9),VEGF expression in the central vascularized regions was minimal.

Normal retinal vascular development in VEGF $F^{164 / 164}$ mice. In $V E G F^{164 / 164}$ mice, the formation of a primitive labyrinth and subsequent remodeling was indistinguishable from that in $V E G F^{+/+}$mice, resulting in similar numbers and sizes of arterioles, venules, and capillaries by P5 (Figure 2k, Table 1). Arterioles grew out over $89 \% \pm 10 \%$, capillaries over $99 \% \pm 5 \%$, and venules 
over $80 \% \pm 12 \%$ of the retinal radius (Table 1). EphrinB2 and SMA expression (not shown), as well as PEC numbers and $\mathrm{EC} / \mathrm{PC}$ ratios (Table 1), hyaloid regression, AC accumulation, and VEGF expression were comparable to $V E G F^{+/+}$at all ages (not shown).

Impaired retinal arterial development in VEGF $188 / 188$ mice. Retinal vessels. In mice expressing only $V_{E G F}$ 188, a normal capillary labyrinth containing PECs (not yet expressing SMA) developed by P3 (not shown). By P5, only half of the normal number of large vessels was present. These were morphologically identified as venules (Figure 3a), did not express ephrin-B2 (Figure 3, b and c), and failed to stain for SMA (Figure 3e), although PECs were present (Figure 3d), which contracted upon fixation (Figure 3f). At P6, ephrin-B2 was detectable in rudimentary retinal arterioles, suggesting that arterial specification was not lost (inset in Figure 3c). At P9, only half of the normal number of arterioles developed, grew out over only $17 \% \pm 13 \%$ of the retina and were smaller in size (Table 1; Figure $3 g$ ). They were also covered by fewer LacZ-positive PECs (Table 1), which stained faintly for SMA at P9 and P14 (Figure 3h). In contrast to the arterial defects, venous and capillary development appeared largely normal. On average, there were five to six venules per retina, which grew out normally (Figure 3, a and g; Table 1), while capillaries grew out over $99 \% \pm 2 \%$ of the retina at P9 (Table 1). The venules were somewhat enlarged and covered by PECs, which were positive for SMA (Figure $3 \mathrm{~h}$ ), possibly due to the arterial underdevelopment and resultant abnormal retinal perfusion. In addition, capillary pruning was decreased, resulting in a more dense capillary bed and a slight increase in the number of PECs per retinal area (Table 1; Figure 3, $d$ and e), but in a normal EC/PC ratio (Table 1 ).

Hyaloid vessels could not be easily dissected away from the retina and had established numerous connections with the retinal vascular network at P5. By P9, several hyaloid vessels had migrated into peripheral retina and established an arterial network (Figure 3g). Hyaloid arteries persisted up to 4 weeks of age (Figure 3i) and penetrated the inner limiting membrane distal to the site of maximal outgrowth of the rudimentary arterioles in the midretina (Figure 3, $\mathrm{i}$ and $\mathrm{j}$ ). Possibly, the hyaloid vessels infiltrated the retina in response to the increased VEGF expression (not shown) that occurred as a result of insufficient arterial supply, thereby salvaging the hypoperfused periphery.

ACs migrated out normally to the retinal periphery by P2 (not shown) and had remodeled into a network that preceded the outgrowing vessels at P5 (Figure 3k).
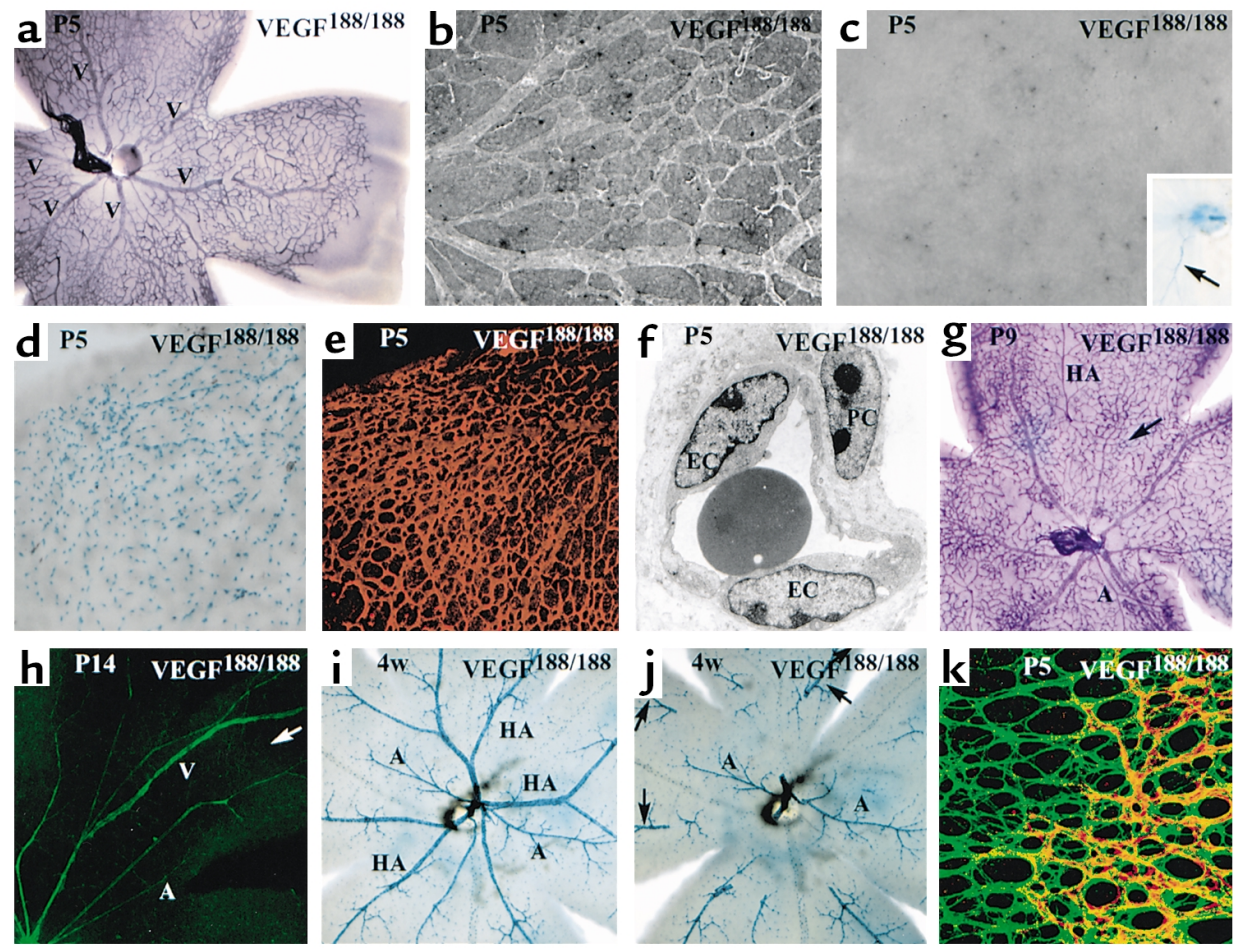

\section{Figure 3}

Retinal vascular development in VEGF ${ }^{188 / 188}$ mice. (a) PECAM staining at P5. Capillary outgrowth comparable to VEGF ${ }^{+/+}$retinas, but no morphologically defined arterioles. (b and c) EB2/CollIV at P5. No EB2 expression at P5. Inset in c: VEGF ${ }^{188 / 188} \times \mathrm{EB}^{\mathrm{LacZ}}$ retina at P6 shows small ephrin-B2-positive vessel (arrow). (d and e) LacZ/Lec/SMA on a VEGF188/188 $\times$ PECLacZ retina at P5, revealing (b) high PC density, (c) but no SMA immunoreactivity. (f) EM at P5 shows normal capillary constriction. (g) PECAM staining at P9 with increased capillary density in the midperipheral retina (arrow) and partially removed hyaloid arteries. (h) SMA immunoreactivity is attenuated in arterioles, but has become positive in venules and capillaries (arrow) at P14. (i and j) VEGF $188 / 188 \times$ PEC LacZ retina at 4 weeks. (i) Persistence of hyaloid arteries and underdevelopment of arterioles. (j) Same retina after dissection of hyaloid arteries. Central part, but not peripheral part (arrows), of hyaloid arteries can be removed. (k) Lec/GFAP. ACs are organized into a tubular network well in front of the vascular bed. 

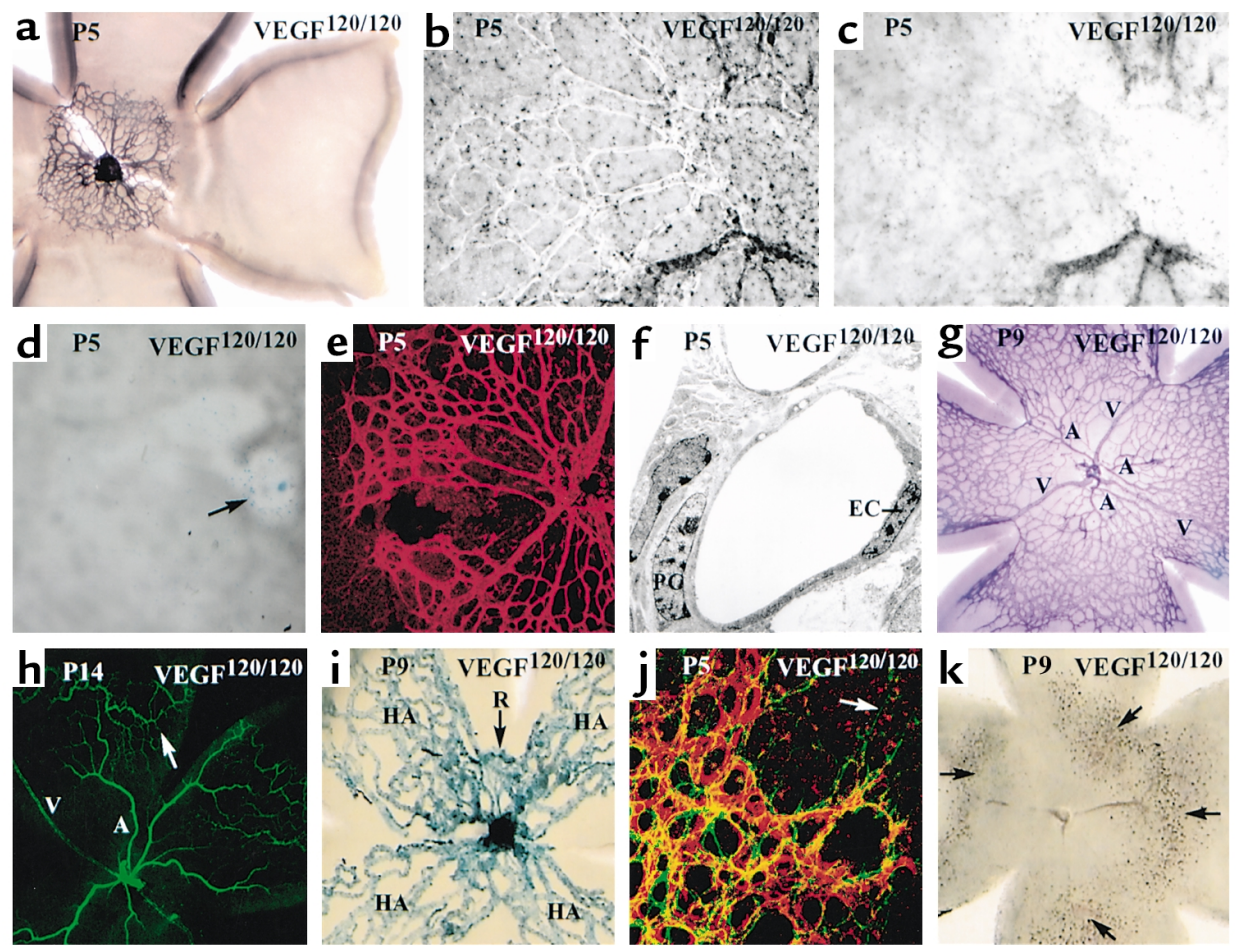

\section{Figure 4}

Retinal vascular development in VEGF120/120 mice. (a) PECAM staining at P5. Dramatic impairment of vascular outgrowth. Arterioles and venules are morphologically indistinguishable. (b and c) EB2/CollIV at P5. Clusters of EB2-positive and -negative vessels alternate. (d and e) LacZ/Lec/SMA on VEGF $120 / 120 \times$ PEC LacZ $^{2}$ retina at P5. (d) Sparse blue cells near optic disc. (e) SMA immunoreactivity is undetectable. (f) EM at P5 shows capillary relaxation. (g) PECAM staining at P9 with more pronounced impairment in arteriolar than venular outgrowth. (h)

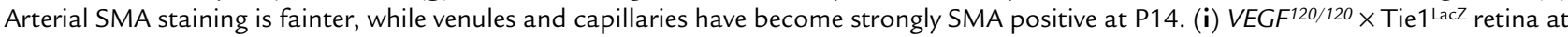
P9, with persisting dilated and tortuous hyaloid arteries. (j) Lec/GFAP. AC organization into a network is present in the vascularized area, but rapidly alters to the primitive bipolar shape and parallel organization in the more peripheral retina (arrow). (k) Unstained whole-mount preparation at P9 with retinal hemorrhages (arrows).

At P9, ACs accumulated in increased numbers at sites where hyaloid vessels made connections to the retinal vascular bed (not shown).

Impaired vascular outgrowth in VEGF ${ }^{120 / 120}$ retinas. Severe retinal vascular defects were detected in VEGF $F^{120 / 120}$ mice. Only a primitive vascular labyrinth of capillarylike vessels with uniform size developed by P5 (Figure 4a). Even though arteries and veins could not be morphologically distinguished, $50 \%$ of the retinal vessels expressed ephrin-B2 (Figure 4, b and c), suggesting that arteriovenous specification occurred before the remodeling stage. PEC recruitment was delayed; only scattered PECs were detected in and around the optic disc (Figure 4d), and retinas were still SMA negative at P5 (Figure 4e). EM illustrated that the sparsity of the PC resulted in a relaxed appearance of capillaries upon fixation (Figure 4f), as in diabetic retinopathy associated with PC dropout.

In contrast to the other genotypes, the capillary bed in VEGF $F^{120 / 120}$ mice covered the retina only over twothirds in the small fraction of VEGF $F^{120 / 120}$ mice that survived until P9 (Table 1). The vascular network had partially remodeled into morphologically recognizable arteries, capillaries, and veins (Figure 4g). To distinguish between a nonspecific general vascular defect and a specific arteriolar defect, outgrowth of arterioles was expressed as a percentage of the radius of the retina as well as the vascular bed. Arterial development was most significantly impaired; less than half of the number of arterioles grew out over only $35 \%$ of the retina and $53 \%$ of the vascular bed (Table 1; Figure 4g). Only half of the number of venules could be morphologically identified in VEGF ${ }^{120 / 120}$ mice at $\mathrm{P} 9$, but venules had a normal lumen size and grew out over only $52 \%$ of the retinal radius, but over $80 \%$ of the vascular bed (Table 1; Figure $4 \mathrm{~g})$. There were more numerous and dilated capillaries in the central retina (Table 1). Fewer PECs surrounded the arterioles, venules, and capillaries, resulting in an increased EC/PC ratio (Table 1 ). The impairment was also reflected by a fragility of the capillaries, causing hemorrhages (Figure 4k). Possibly because of the increased hemodynamic stress, these PECs stained somewhat more strongly for SMA (Figure 4h). VEGF expression was markedly upregulated in the avascular retina, suggesting that the peripheral retina was highly ischemic (not shown).

No signs of hyaloid regression were observed in VEGF ${ }^{120 / 120}$ mice at P9, and the hyaloid vessels appeared dilated and tortuous (Figure 4i). However, unlike in VEGF ${ }^{188 / 188}$ mice, the hyaloid arteries in 
$V_{E G F}^{120 / 120}$ mice neither penetrated the retina nor made connections with the retinal vasculature.

ACs initially migrated normally out to the periphery of the retina (not shown). By P5 and P9, ACs in the central vascularized retina associated with retinal vessels showed strong GFAP expression. In the avascular retinal periphery, however, GFAP immunoreactivity was weak (Figure 4j), possibly due to tissue damage secondary to the ischemia. VEGF expression was more abundant at the edge of the vascular bed, likely the result of local hypoxia (not shown).

Expression of VEGF receptors. Whole-mount in situ hybridization of retinas from $V E G F^{+/+}$pups at P5 and P9 revealed that NP-1 was expressed in retinal arterioles and, at a much lower level, in retinal venules (Figure 5a). Higher magnification suggested that NP-1 is expressed in ECs (Figure 5b) and likely also in PECs (Figure 5, b and $\mathrm{c}$ ), which were also labeled for PDGF receptor- $\beta$ (Figure 5d). NP-1 mRNA expression has been reported previously in ECs and PECs (16). By triple immunofluorescent staining for NP-1, lectin, and SMA, NP-1 was detectable in retinal neurons, but not in ECs or PECs in the retina (Figure 5, e and $\mathrm{f}$ ). Immunostaining for VEGFR-1 or VEGFR-2 indicated that VEGFR-1 was expressed in ECs and vSMCs of arteries and veins (Figure 5 , $g$ and $h$ ), whereas VEGF-R2 was expressed only in ECs of capillaries and venules in the retina (Figure 5, $\mathrm{i}$ and j), brain, heart, and kidney (not shown). Specificity of the VEGFR-1 and VEGFR-2 staining has been reported elsewhere (17). Expression of VEGFR-1 (18) and NP-1 (19) has been documented previously in PECs.

Arterial development in other organs. A similar number of glomerular arterioles was present in anti-SMA-stained sections of $V E G F^{+/+}, V E G F^{164 / 164}$, and $V E G F^{188 / 188}$ kidneys (arterioles per kidney section: $45 \pm 2,49 \pm 9$, and $42 \pm 7$, respectively; $n=5 ; P=\mathrm{NS}$ ). Comparable densities of small, medium, and large coronaries were found in $V E G F^{+/+}$(vessels per square millimeter: $8.5 \pm 2.9,4.3 \pm 1.0$, and $3.4 \pm 1.6 ; n=6$ ), $V E G F^{164 / 164}$ (vessels per square millimeter: $8.1 \pm 3.1,4.2 \pm 2.0$, and $3.5 \pm 1.8 ; n=4, P=\mathrm{NS})$, and $V E G F^{188 / 188}$ hearts (vessels per square millimeter: $9.6 \pm 2.8,6.2 \pm 2.6$, and $2.5 \pm 1.7 ; n=6, P=\mathrm{NS}) . V E G F^{120 / 120}$ mice had fewer renal and coronary arteries (6).

\section{Discussion}

The present study investigates the distinct role of the different VEGF isoforms in retinal vascular development. Retinal vascular development was normal in $V E G F^{164 / 164}$ mice, indicating that this isoform contains all necessary information for normal outgrowth and remodeling of blood vessels. In contrast, $V E G F^{120 / 120}$ mice exhibited severe vascular defects, with impaired venous and severely defective arterial vascular development in the retina. VEGF $F^{188 / 188}$ mice had normal venous development, but aborted arterial outgrowth.

Role of the VEGF isoforms in endothelial outgrowth. Astrocytes migrate in front of ECs and guide them to the periphery of the retina by releasing VEGF (20). Outgrowth of the vascular bed was normal in $V E G F^{164 / 164}$ and $V E G F^{188 / 188}$ mice (except for the arterioles in $V E G F^{188 / 188}$ retinas). In contrast, in VEGF ${ }^{120 / 120}$ mice there was a defect in EC outgrowth, affecting all vessel types. This may be due to the fact that $\mathrm{VEGF}_{120}$ is less potent in inducing EC mitosis (21) or has reduced affinity for VEGFR-1 (22), which could affect EC function or survival (6). Alternatively, $V E_{164}$ and

\begin{abstract}
Figure 5
VEGF receptor expression in $\mathrm{VEGF}^{+/+}$retinas. (a) Whole-mount in situ hybridization for NP-1 at P5. Expression is prominent in arterioles and discrete in venules. (b) In situ hybridization on retinal sections at P7 is suggestive of NP-1 expression in ECs as well as PECs (arrows). ( $\mathbf{c}$ and $\mathbf{d}$ ) High magnification of whole-mount in situ hybridization for (c) NP-1 and (d) PDGF receptor- $\beta$ (PDGFR $\beta$ ) illustrates that PECs are positive for PDGFR $\beta$ and suggest NP-1 expression in PECs (arrows). (e and j) Fluorescent triple immunostaining using lectin, anti-SMA, and anti-VEGFR on sections from $V E G F^{+/+}$eyes at $\mathrm{P} 9$. Lectin (blue), VEGFR (red), and SMA (green). (e and $\mathbf{f}$ ) NP-1 is detectable in retinal neurons (arrowheads), but its expression is beyond the detection limit in vessels by immunostaining. ( $g$ and $\mathbf{h}$ ) VEGFR-1 (R1) is expressed in vSMCs as well as ECs (arrow). (i and $\mathbf{j})$ VEGFR-2 (R2) expression is only detectable in the ECs (arrows) in capillaries and venules.
\end{abstract}
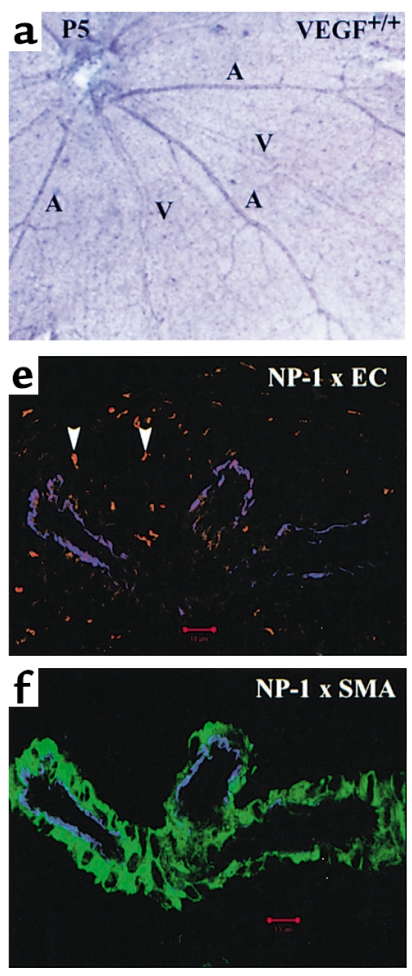
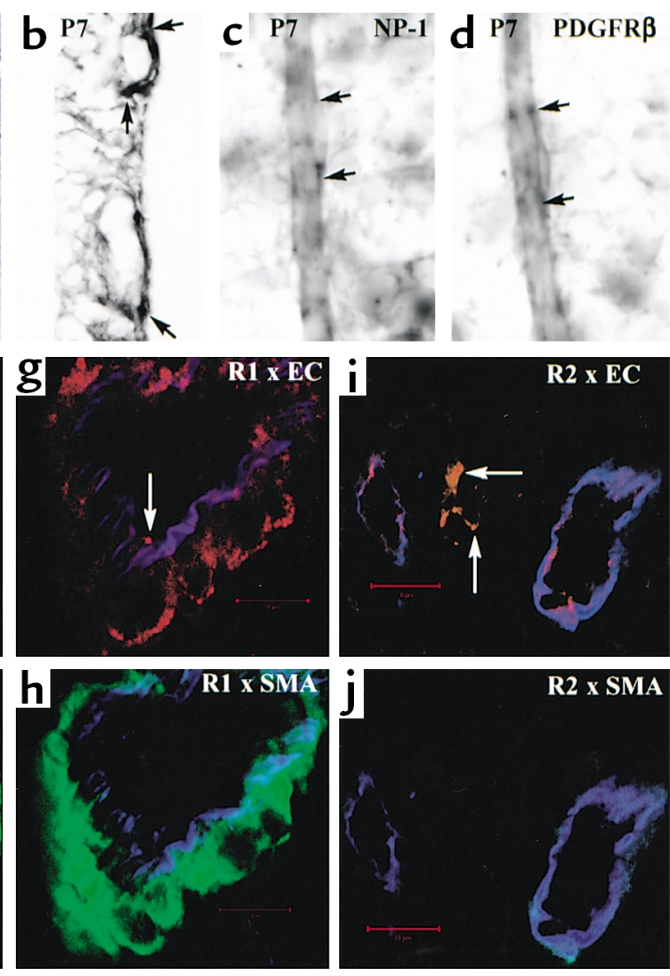
$\mathrm{VEGF}_{188}$, in binding the extracellular matrix, may provide matrix-associated guidance cues that facilitate EC migration through the retina, possibly through the establishment of a VEGF gradient or "trail" along which ECs migrate. Such cues would likely be absent in $V E G F^{120 / 120}$ mice in which diffusion of the soluble VEGF $_{120}$ isoform would induce random EC migration. These hypotheses are consistent with the finding that VEGF $_{120}$-expressing tumors are hypovascular, in contrast to $\mathrm{VEGF}_{164}$ - or $\mathrm{VEGF}_{188}$-expressing tumors (23). This would implicate an important role for the different VEGF isoforms in correct patterning of nascent vessels. Vessel patterning is an essential characteristic of normal angiogenesis, which remains poorly understood at the molecular level.

Role of the VEGF isoforms in retinal arterial development. Genotype-specific defects were observed in the formation of retinal arterioles and venules. In $V E G F^{+/+}$and $V E G F^{164 / 164}$ mice, arterioles reached out over approximately $90 \%$ of the entire vascular bed at P9. In $V E G F^{120 / 120}$ mice, there were fewer and smaller venules and arterioles but, whereas venules reached out over $80 \%$, arterioles extended over only approximately $50 \%$ of the vascular bed. Arteriolar defects were even more pronounced in $V E G F^{188 / 188}$ mice, in which arterioles (but not venules) were smaller and extended over only $18 \%$ of the vascular bed. Thus, the $\mathrm{VEGF}_{164}$ isoform is sufficient for venular and arteriolar development, the VEGF $_{188}$ isoform allows venular but not arteriolar development, and the VEGF ${ }_{120}$ isoform is insufficient for venular, but especially for arteriolar, development.

Arterial EC specification. The current hypothesis suggests that "arterialization" of an undifferentiated vascular network requires remodeling and recruitment of vSMCs in response to hemodynamic forces (reviewed by ref. 24). More recent studies proposed that arterial and venous ECs are molecularly distinct from the earliest stages of vascular development, distinguishable by their expression of gridlock (25), D114 (26), Bmx (27), NP-1 (16), ephrin-B2 (on arterial ECs) and its receptor Eph-B4 (on venous ECs) (7), and activin receptor-like kinase-1 (28). Consistent with this revised model, ephrin-B2 was expressed in half of the vessels in $V E G F^{+/+}$and $V E G F^{164 / 164}$ mice, even before arterioles could be morphologically distinguished. Since ephrin-B2 was also expressed in unremodeled retinal vessels in $V E G F^{120 / 120}$ mice, the impaired arteriolar development was not attributable to an aborted arteriolar EC specification. In contrast, ephrin-B2 expression in $V E G F^{188 / 188}$ mice was delayed until P6, when rudimentary arterioles began to grow out, indicating that these ECs were arterially differentiated but failed to grow out normally. Whether defective arterial outgrowth in $V E G F^{188 / 188}$ mice resulted from delayed or impaired arterial specification of EC precursors and/or outgrowth of arterial ECs, and why the $V \mathrm{VGF}_{120}$ and $\mathrm{VEGF}_{164}$ isoforms are more efficient than $V_{E G F}$ in inducing arterial specification and/or outgrowth, remain to be determined. Intrigu- ing possibilities are that the short isoforms induce expression of arterial markers (as demonstrated for Bmx) (27) or affect angioblasts even at an earlier stage or that the $\mathrm{VEGF}_{188}$ isoform might be unable to provide spatial guidance or differentiation cues for retinal arteriolar ECs because it remains associated with the extracellular matrix.

$P E C$ recruitment. PEC recruitment was normal in $V E G F^{164 / 164}$ mice. In contrast, $V E G F^{120 / 120}$ mice exhibited generally impaired PEC recruitment resulting in capillary dilatation and extravasation of red blood cells, as described in the hearts of these mice (6), as well as in other conditions of PEC dysfunction or dropout (29). ECs release signals (such as PDGF-BB) essential for PC recruitment (10). Since expression of PDGF-BB is reduced in VEGF ${ }^{120 / 120}$ mice (6), it is possible that the impairment of PEC recruitment is attributable to the general endothelial defects in VEGF ${ }^{120 / 120}$ mice. Interestingly, VEGFR-1 was expressed in retinal (as well as renal, brain, and cardiac) arteriolar and venular PECs (this study and ref. 18), indicating that VEGF may have direct effects on PC recruitment (10). Since the $\mathrm{VEGF}_{120}$ isoform binds with a lower affinity to VEGFR-1 than the VEGF $_{164}$ isoform (22), this short isoform may be unable to recruit PECs as efficiently as the other isoforms. $V E G F^{188 / 188}$ mice, on the other hand, showed normal PEC recruitment to venules and capillaries, but reduced coverage of underdeveloped retinal arterioles. This may again suggest that the impaired PC recruitment is secondary to abnormal EC function in the underdeveloped arterioles. Alternatively, diffusion of the smaller VEGF isoforms may be essential for chemoattracting VEGFR-1-positive PECs. The specific defect of PC recruitment around arterioles may also suggest an essential interaction of the $\mathrm{VEGF}_{164}$ isoform with NP-1, which is more abundantly expressed in retinal arterioles than in venules. Although not yet described, the $\mathrm{VEGF}_{188}$ isoform likely binds to NP-1 since it contains exon 7 , encoding the essential amino acid residues for such interaction (30). Perhaps this long isoform (which binds to the matrix) is unable to reach the NP-1 receptor on PECs or impairs PC migration because it immobilizes the NP-1-positive PCs at the site of $\mathrm{VEGF}_{188}$ production. A role for NP-1 in cell adhesion has been suggested previously, although the nature of the ligand remains unknown (31). Another possibility is that blood flow in the underdeveloped arterioles is abnormal, which could impair their maturation. Finally, the retinal phenotype may have been influenced by the impaired angiogenesis in the heart, bone, and lungs of $V E G F^{120 / 120}$ mice $(6,32,33)$, the growth retardation in the $V E G F^{188 / 188}$ mice, or the relative overexpression of a single isoform. Indeed, each of these knock-in mice expressed a single isoform at a level comparable to the total expression of the three isoforms in $V E G F^{+/+}$mice (this study and ref. 6). However, it is unlikely that the retinal phenotype was only secondary to impaired vascular growth in other 
organs, because no such specific arterial defects were detected in other organs.

Arterial development in other organs. While it may be a surprise that this long isoform has a restricted role in establishing arterioles in the retina, emerging evidence indicates that the mechanisms of vessel formation (34), as well as the differential expression of the VEGF isoforms (32), are highly dependent on the tissue microenvironment. Noteworthy in this context is that retinal PECs are derived from neural crest cells, whereas PECs in other tissues are derived from other sources (35).

Role of VEGF isoforms in the regression of byaloid vessels. In $V E G F^{188 / 188}$ mice, the retinal arteriolar underdevelopment prompted ingrowth by hyaloid arteries, which established connections with the retinal vascular network. In contrast, hyaloid vessels in VEGF ${ }^{120 / 120}$ mice did not enter the retina, but were dilated and tortuous. The dissimilarity in hyaloid vessel phenotype may be attributable to the difference in solubility between the VEGF isoforms. VEGF 188 is matrix bound and presumably remains associated within the retina. Since hyaloid vessels lie closely apposed to the retina, the increased $\mathrm{VEGF}_{188}$ levels might attract these hyaloid vessels into the ischemic peripheral retina (Figure 1g). Alternatively, $\mathrm{VEGF}_{188}$ can be proteolytically cleaved into a shorter, soluble isoform, which would establish a VEGF gradient attracting hyaloid vessels (36). Soluble VEGF 120 would be expected to diffuse into the vitreous cavity (Figure 1f), explaining why hyaloid vessels persisted, became dilated and tortuous, and failed to enter the retina. Whether binding of $\mathrm{VEGF}_{188}$ to NP-1 mediates adhesion of hyaloid vessels to the retina remains to be determined.

The persistence of hyaloid vessels in mice lacking the $\mathrm{VEGF}_{164}$ isoform resembles the persistent hyperplastic primary vitreous disorder in humans, a common blinding congenital anomaly resulting from a failure of hyaloid vessel regression (37). Taken together, these findings suggest a novel role for the various VEGF isoforms in endothelial outgrowth and arterial development.

\section{Acknowledgments}

The authors thank A. Van Lommel (Pathology Department, Catholic University Leuven) and A. Bouché, K. Vandevelde, W. Man, L. Kieckens, A. Manderveld, F. De Wever, K. Maris, T. Vancoetsem, E. Gils, K. Bijnens, A. Vandenhoeck, P. Van Wesemael, L. Godde, S. Lucas, C. Van Huylebroeck, S. Wyns (The Center for Transgene Technology and Gene Therapy, Leuven) for assistance. They acknowledge K. Alitalo for providing the Tie1 promotor. I. Stalmans is a Research Assistant of the Fonds voor Wetenschappelijk Onderzoek (FWO), Belgium and P. D'Amore is a Jules and Doris Stein Research to Prevent Blindness Professor. This work is further supported by grants from the FWO (G012500), the Geconcerteerde Onderzoeksacties Belgium (2001/09), and the European Union (BMH4-CT98-3380) to P. Carmeliet; from the
NIH (HL-6221 and CA-45548) to D.J. Anderson and P. D'Amore; and from Deutsche Forschungsgemeinschaft to H.-P. Hammes.

1. Carmeliet, P., et al. 1996. Abnormal blood vessel development and lethality in embryos lacking a single VEGF allele. Nature. 380:435-439.

2. Carmeliet, P., and Jain, R.K. 2000. Angiogenesis in cancer and other diseases. Nature. 407:249-257.

3. Neufeld, G., Cohen, T., Gengrinovitch, S., and Poltorak, Z. 1999. Vascular endothelial growth factor (VEGF) and its receptors. FASEB J. 13:9-22.

4. Miao, H.Q., and Klagsbrun, M. 2000. Neuropilin is a mediator of angiogenesis. Cancer Metastasis Rev. 19:29-37.

5. Soker, S., Takashima, S., Miao, H.Q., Neufeld, G., and Klagsbrun, M. 1998. Neuropilin-1 is expressed by endothelial and tumor cells as an isoform-specific receptor for vascular endothelial growth factor. Cell. 92:735-745.

6. Carmeliet, P., et al. 1999. Impaired myocardial angiogenesis and ischemic cardiomyopathy in mice lacking the vascular endothelial growth factor isoforms VEGF164 and VEGF188. Nat. Med. 5:495-502.

7. Wang, H.U., Chen, Z.F., and Anderson, D.J. 1998. Molecular distinction and angiogenic interaction between embryonic arteries and veins revealed by ephrin-B2 and its receptor Eph-B4. Cell. 93:741-753.

8. Korhonen, J., et al. 1995. Endothelial-specific gene expression directed by the tie gene promoter in vivo. Blood. 86:1828-1835.

9. Tidhar, A., et al. 2001. A novel transgenic marker for migrating limb muscle precursors and for vascular smooth muscle cells. Dev. Dyn. 220:60-73.

10. Benjamin, L.E., Hemo, I., and Keshet, E. 1998. A plasticity window for blood vessel remodelling is defined by pericyte coverage of the preformed endothelial network and is regulated by PDGF- B and VEGF. Development. 125:1591-1598.

11. Kawakami, A., Kitsukawa, T., Takagi, S., and Fujisawa, H. 1996. Developmentally regulated expression of a cell surface protein, neuropilin, in the mouse nervous system. J. Neurobiol. 29:1-17.

12. Bergemann, A.D., Cheng, H.J., Brambilla, R., Klein, R., and Flanagan, J.G. 1995. ELF-2, a new member of the Eph ligand family, is segmentally expressed in mouse embryos in the region of the hindbrain and newly forming somites. Mol. Cell. Biol. 15:4921-4929.

13. Lindahl, P., Johansson, B.R., Leveen, P., and Betsholtz, C. 1997. Pericyte loss and microaneurysm formation in PDGF-B-deficient mice. Science. 277:242-245.

14. Hammes, H.P., et al. 1996. Acceleration of experimental diabetic retinopathy in the rat by omega-3 fatty acids. Diabetologia. 39:251-255.

15. De Schaepdrijver, L., Simoens, P., Lauwers, H., De Geest, J.P., and Charlier, G. 1989. The hyaloid vascular system of the pig. A light and scanning electron microscopic study. Anat. Embryol. 180:549-554.

16. Moyon, D., Pardanaud, L., Yuan, L., Bréant, C., and Eichmann, A. 2001. Plasticity of endothelial cells during arterial-venous differentiation in the avian embryo. Development. 128:3359-3370.

17. Carmeliet, P., et al. 2001. Synergism between vascular endothelial growth factor and placental growth factor contributes to angiogenesis and plasma extravasation in pathological conditions. Nat. Med. 7:575-583

18. Suzuma, K., et al. 2000. Vascular endothelial growth factor induces expression of connective tissue growth factor via KDR, Flt1, and phosphatidylinositol 3-kinase-akt-dependent pathways in retinal vascular cells. J. Biol. Chem. 275:40725-40731.

19. Ishida, A., et al. 2001. Expression of vascular endothelial growth factor receptors in smooth muscle cells. J. Cell. Physiol. 188:359-368.

20. Stone, J., et al. 1995. Development of retinal vasculature is mediated by hypoxia-induced vascular endothelial growth factor (VEGF) expression by neuroglia. J. Neurosci. 15:4738-4747.

21. Keyt, B.A., et al. 1996. The carboxyl-terminal domain (111-165) of vascular endothelial growth factor is critical for its mitogenic potency. J. Biol. Chem. 271:7788-7795.

22. Gitay-Goren, H., et al. 1996. Selective binding of VEGF121 to one of the three vascular endothelial growth factor receptors of vascular endothelial cells. J. Biol. Chem. 271:5519-5523.

23. Grunstein, J., Masbad, J.J., Hickey, R., Giordano, F., and Johnson, R.S. 2000. Isoforms of vascular endothelial growth factor act in a coordinate fashion to recruit and expand tumor vasculature. Mol. Cell. Biol. 20:7282-7291.

24. Yancopoulos, G.D., Klagsbrun, M., and Folkman, J. 1998. Vasculogenesis, angiogenesis, and growth factors: ephrins enter the fray at the border. Cell. 93:661-664.

25. Zhong, T.P., Rosenberg, M., Mohideen, M.A., Weinstein, B., and Fishman, M.C. 2000. Gridlock, an HLH gene required for assembly of the aorta in zebrafish. Science. 287:1820-1824.

26. Shutter, J.R., et al. 2000. Dll4, a novel Notch ligand expressed in arte- 
rial endothelium. Genes. Dev. 14:1313-1318.

27. Rajantie, I., et al. 2001. Bmx tyrosine kinase has a redundant function downstream of angiopoietin and vascular endothelial growth factor receptors in arterial endothelium. Mol. Cell. Biol. 21:4647-4655.

28. Urness, L.D., Sorensen, L.K., and Li, D.Y. 2000. Arteriovenous malformations in mice lacking activin receptor-like kinase-1. Nat. Genet. 26:328-331.

29. Lindahl, P., Hellstrom, M., Kalen, M., and Betsholtz, C. 1998. Endothelial-perivascular cell signaling in vascular development: lessons from knockout mice. Curr. Opin. Lipidol. 9:407-411.

30. Gitay-Goren, H., Soker, S., Vlodavsky, I., and Neufeld, G. 1992. The binding of vascular endothelial growth factor to its receptors is dependent on cell surface-associated heparin-like molecules. J. Biol. Chem. 267:6093-6098.

31. Shimizu, M., Murakami, Y., Suto, F., and Fujisawa, H. 2000. Determination of cell adhesion sites of neuropilin-1. J. Cell. Biol. 148:1283-1293.
32. Ng, Y.S., Rohan, R., Sunday, M.E., Demello, D.E., and D’Amore, P.A. 2001. Differential expression of VEGF isoforms in mouse during development and in the adult. Dev. Dyn. 220:112-121.

33. Maes, C., et al. 2002. Impaired angiogenesis and enchondral bone formation in mice lacking the vascular endothelial growth factor isoforms VEGF164 and VEGF188. Dev. Dyn. In press.

34. Lecouter, J., et al. 2001. Identification of an angiogenic mitogen selective for endocrine gland endothelium. Nature. 412:877-884.

35. Etchevers, H.C., Vincent, C., Le Douarin, N.M., and Couly, G.F. 2001 The cephalic neural crest provides pericytes and smooth muscle cells to all blood vessels of the face and forebrain. Development. 128:1059-1068.

36. Plouet, J., et al. 1997. Extracellular cleavage of the vascular endothelial growth factor 189-amino acid form by urokinase is required for its mitogenic effect. J. Biol. Chem. 272:13390-13396.

37. Silbert, M., and Gurwood, A.S. 2000. Persistent hyperplastic primary vitreous. Clin. Eye Vis. Care. 12:131-137. 